\title{
Warm Inflation with Nonminimal Derivative Coupling
}

\author{
Kourosh Nozari, M. Shoukrani, and N. Rashidi \\ Department of Physics, Faculty of Basic Sciences, University of Mazandaran, P.O. Box 47416-1467, Babolsar, Iran \\ Correspondence should be addressed to Kourosh Nozari; knozari@umz.ac.ir
}

Received 11 January 2014; Revised 8 March 2014; Accepted 9 March 2014; Published 27 April 2014

Academic Editor: Kingman Cheung

Copyright (C) 2014 Kourosh Nozari et al. This is an open access article distributed under the Creative Commons Attribution License, which permits unrestricted use, distribution, and reproduction in any medium, provided the original work is properly cited. The publication of this article was funded by SCOAP ${ }^{3}$.

\begin{abstract}
We study the effects of the nonminimal derivative coupling on the dissipative dynamics of the warm inflation where the scalar field is nonminimally coupled to gravity via its kinetic term. We present a detailed calculation of the cosmological perturbations in this setup. We use the recent observational data from the joint data set of WMAP9 $+\mathrm{BAO}+\mathrm{H}_{0}$ and also the Planck satellite data to constrain our model parameters for natural and chaotic inflation potentials. We study also the levels of non-Gaussianity in this warm inflation model and we confront the result with recent observational data from the Planck satellite.
\end{abstract}

\section{Introduction}

Although the hot big bang scenario has considerable successes in explaining the general properties of our universe, this model cannot address properly some issues such as flatness, horizon, and relics problems. The latest observations show that a period of accelerating expansion (inflation) during the early history of the universe with $\ddot{a}>0$ $(p<-\rho / 3)$ can solve these problems. Inflation also provides the primordial perturbations which seed the formation of structure in the universe [1]. Despite the great successes of the inflation paradigm, there is a problem for realization of this scenario that we do not know how to integrate it with ideas of particle physics. Also problems such as unexpected low power spectrum at large scales and egregious running of the spectral index [2] are some yet unsolved problems in this paradigm. From a thermodynamic viewpoint, there are two different dynamical realizations of inflation which are called cold and warm inflation. In the standard inflationary model, called supercooled or isentropic inflation, the universe rapidly supercools during the inflation and its temperature decreases, which is a result of the exponential expansion. When the inflation ends, a reheating phase occurs, which explains the subsequent processes and evolution. The fluctuations created during inflation are zero-point ground state fluctuations and evolution of the inflaton field is governed by the ground state evolution equations. The other picture of inflation dynamics is the warm or nonisentropic inflation [3-5]. In this picture, the dissipative effects are significant. So, that radiation production and inflationary expansion occur concurrently. Several mechanisms for implementing such a dissipation during inflation have been proposed $[6,7]$. In warm inflationary models, the sources of density fluctuations are the thermal fluctuations. Matter fields interact with particles that are in a thermal bath with mean temperature smaller than the grand unified theories (GUT) critical temperature [8]. The most important candidate for the inflaton is the Standard Model's Higgs field. Nevertheless, if Higgs field is minimally coupled to gravity, it cannot realize a good inflation stage [9]. To preserve the Higgs field as an inflaton candidate, a model has been postulated in which the Higgs field is nonminimally coupled to gravity [10]. It has been argued in [11, 12] that this nonminimal coupling breaks the unitary bound by giving rise to nonrenormalizabe operators. However, the authors in [13] have shown that this is not actually the case and the unitary bound is preserved in this setup. On the other hand, if gravity is nonminimally coupled to derivatives of the scalar field, the unitary bound is preserved during inflation. So, this model can explain the inflationary phase properly $[14,15]$. In recent years, the issue of inflation and also the late time accelerated expansion with nonminimal derivative coupling are studied in detail [16-20]. Nevertheless, the warm inflation 
dynamics in the context of nonminimal derivative coupling and perturbations in this setup have not been studied yet. Therefore, the present study is devoted to filling this gap.

With these preliminaries, the goal of the present study is to investigate the effects of the nonminimal derivative coupling on the dissipative dynamics of the warm inflation. We investigate the effects of the nonminimal derivative coupling and dissipation on the inflationary dynamics of the model. Then, we present a detailed calculation of the perturbations in this framework. By using the recent observational data from WMAP9 $+\mathrm{BAO}+\mathrm{H}_{0}$ and also the Planck satellite data, we constrain our model parameters for natural and chaotic inflation potentials. Also we study the levels of nonGaussianity in this scenario and we confront the obtained results with the recent observational data from the Planck satellite.

\section{The Setup}

The action of a 4-dimensional model in the presence of the nonminimal derivative coupling between the scalar field and gravity is given by

$$
\begin{aligned}
S=\int \sqrt{-g} d^{4} x( & \frac{M_{p}^{2} R}{2}-\frac{1}{2} g^{\mu \nu} \partial_{\mu} \varphi \partial_{\nu} \varphi \\
& \left.+\frac{1}{2 M^{2}} G^{\mu \nu} \partial_{\mu} \varphi \partial_{\nu} \varphi-V(\varphi)\right),
\end{aligned}
$$

where $G^{\mu \nu}=R^{\mu \nu}-(1 / 2) R g^{\mu \nu}$ is the Einstein tensor and $M$ is a constant having the dimension of mass and $M_{p}=2.4 \times$ $10^{18} \mathrm{GeV}$ is the reduced Planck mass. $R$ is the Ricci scalar and $V(\varphi)$ is the potential of the scalar field $\varphi$. Variation of action (1) with respect to the scalar field gives the following equation of motion:

$$
\left(1+3 \frac{H^{2}}{M^{2}}\right) \ddot{\varphi}+3 H\left(1+3 \frac{H^{2}}{M^{2}}+2 \frac{\dot{H}}{M^{2}}\right) \dot{\varphi}+V^{\prime}(\varphi)=0 .
$$

If we consider phenomenologically a dissipation coefficient $\Gamma$ that is responsible for the decay of the scalar field into the radiation during the inflationary regime, the scalar field equation of motion will be as follows:

$$
\left(1+3 \frac{H^{2}}{M^{2}}\right) \ddot{\varphi}+3 H\left(1+3 \frac{H^{2}}{M^{2}}+2 \frac{\dot{H}}{M^{2}}\right) \dot{\varphi}+V^{\prime}(\varphi)=-\Gamma \dot{\varphi}
$$

$\Gamma$ can be assumed to be a constant or a function of the scalar field $\varphi$ or the temperature $T$ or both of them. In the slow-roll approximation where $\ddot{\varphi} \ll|(3 H+\Gamma) \dot{\varphi}|$ and $|\dot{H}| \ll H^{2}$, the scalar field equation of motion takes the following form:

$$
\dot{\varphi}=-\frac{V^{\prime}(\varphi)}{\left(\Gamma+3 H\left(1+3 H^{2} / M^{2}\right)\right)} .
$$

Note that the sufficient condition for canonical normalization of the scalar field is given by $H^{2} \gg M^{2}[14,15]$. The energy density and the pressure of the scalar field are given by

$$
\begin{aligned}
& \rho_{\varphi}=\left(1+9 \frac{H^{2}}{M^{2}}\right) \frac{\dot{\varphi}^{2}}{2}+V(\varphi), \\
& P_{\varphi}=\frac{\dot{\varphi}^{2}}{2}-V(\varphi)-\frac{1}{2 M^{2}}\left(3 H^{2}+2 \dot{H}\right) \dot{\varphi}^{2}-2 \frac{H}{M^{2}} \dot{\varphi} \ddot{\varphi} .
\end{aligned}
$$

Also, the energy conservation equation of the model is given by

$$
\dot{\rho}_{\varphi}+3 H\left(\rho_{\varphi}+P_{\varphi}\right)=-\Gamma \dot{\varphi}^{2}
$$

During the inflaton evolution, the dissipation effect leads to the production of entropy. The entropy density of the radiation $S(\varphi, T)$ in this nonminimal setup is defined by $S=$ $-\int(d V(\varphi) / T)$. Now the total energy density of the system is given by

$$
\rho=\left(1+9 \frac{H^{2}}{M^{2}}\right) \frac{\dot{\varphi}^{2}}{2}+V(\varphi)+T S
$$

and using the relation (6), the rate of entropy production can be deduced. In this setup, entropy production is affected by the nonminimal derivative coupling between the scalar field and gravity. This can be seen more explicitly in the following relation:

$$
T(\dot{S}+3 H S)=\Gamma \dot{\varphi}^{2}
$$

where by (4), $\dot{\varphi}$ is directly related to the nonminimal derivative coupling. The basic idea of the warm inflation is that radiation production is occurring concurrently with inflationary expansion due to dissipation from the inflaton field system. The equation of state for radiation is given by $P_{\gamma}=\rho_{\gamma} / 3$. Therefore, the continuity equation for the radiation yields the following result:

$$
\dot{\rho}_{R}+4 H \rho_{R}=\Gamma \dot{\varphi}^{2} \text {. }
$$

We assume a quasi-stable radiation production during the warm inflation phase $[21,22]$; that is, $\dot{\rho}_{R} \ll 4 H \rho_{R}$ and $\dot{\rho}_{R} \ll$ $\Gamma \dot{\varphi}^{2}$. So we can write

$$
\rho_{R}=\frac{\Gamma}{4 H} \dot{\varphi}^{2}
$$

Substituting (4) into this relation, we get

$$
\rho_{R}=\frac{\Gamma V^{\prime 2}(\varphi)}{4 H\left(\Gamma+3 H\left(1+3 H^{2} / M^{2}\right)\right)^{2}} .
$$

Now we define the dissipation factor as follows:

$$
Q \equiv \frac{\Gamma}{3 H}
$$


which is a dimensionless parameter. With this definition, we can write

$$
\dot{\varphi}=-\frac{V^{\prime}(\varphi)}{3 H\left(Q+\left(1+3 H^{2} / M^{2}\right)\right)} .
$$

During the slow-roll stage, the scalar field evolution is damped. For the high (or weak) dissipation regime we have $Q \gg 1$ (or $Q \ll 1)$, respectively.

Now, in the spatially flat FRW background, the Friedmann equation can be written as follows:

$$
H^{2}=\frac{1}{3 M_{p}^{2}}\left[\left(1+9 \frac{H^{2}}{M^{2}}\right) \frac{\dot{\varphi}^{2}}{2}+V(\varphi)\right] .
$$

The slow-roll regime can be parametrized by a set of slowroll parameters $\varepsilon, \eta$, and $\beta$, defined by

$$
\varepsilon \equiv-\frac{\dot{H}}{H^{2}}, \quad \eta \equiv-\frac{\ddot{H}}{H \dot{H}}, \quad \beta \equiv 2 \varepsilon \eta-\frac{d \eta}{d t} .
$$

Inflation can be realized only if $\{\varepsilon, \eta, \beta\}<1$; once one of these parameters reaches the unity, the inflation phase terminates. We need to calculate these parameters in our nonminimal derivative coupling model. For $\varepsilon$ we get

$$
\varepsilon=\frac{\left(V^{\prime}(\varphi)\right)^{2}}{6 M_{p}^{2} H^{3}\left(\Gamma+3 H\left(1+3 H^{2} / M^{2}\right)\right)},
$$

and the second slow-roll parameter $\eta$ becomes

$$
\begin{aligned}
& \eta= \frac{2 V^{\prime \prime}(\varphi)}{H\left(\Gamma+3 H\left(1+3 H^{2} / M^{2}\right)\right)} \\
&-\left(V ^ { \prime } ( \varphi ) \left(6 H\left(1+\frac{3 H^{2}}{M^{2}}\right) H^{\prime}\right.\right. \\
&\left.\left.\quad+\frac{18 H^{3} H^{\prime}}{M^{2}}+\Gamma^{\prime} H+H^{\prime} \Gamma\right)\right) \\
& \\
& \quad \times\left(H^{2}\left(\Gamma+3 H\left(1+\frac{3 H^{2}}{M^{2}}\right)\right)^{2}\right)^{-1} .
\end{aligned}
$$

And finally, the third slow-roll parameter takes the following form:

$$
\begin{aligned}
\beta= & 2 \varepsilon \eta+\frac{2 V^{\prime}(\varphi) V^{\prime \prime \prime}(\varphi)}{H\left(\Gamma+3 H\left(1+3 H^{2} / M^{2}\right)\right)} \\
& +\frac{V^{\prime}(\varphi)\left(36\left(H^{3} / M^{2}\right) H^{\prime}+6 H H^{\prime}+\Gamma^{\prime} H+H^{\prime} \Gamma\right)}{H\left(\Gamma+3 H\left(1+3 H^{2} / M^{2}\right)\right)^{2}} \\
& \times\left[\frac{4 V^{\prime \prime}(\varphi)}{H\left(\Gamma+3 H\left(1+3 H^{2} / M^{2}\right)\right)}+2 \eta\right]
\end{aligned}
$$

$$
\begin{aligned}
& -\varepsilon H\left(108 H^{2} H^{\prime 2}+36 \frac{H^{3}}{M^{2}} H^{\prime \prime}+\Gamma^{\prime \prime} H\right. \\
& \left.+2 H^{\prime} \Gamma^{\prime}+H^{\prime \prime} \Gamma+6 H H^{\prime \prime}+6 H^{\prime 2}\right) \\
& \times\left(\Gamma+3 H\left(1+\frac{3 H^{2}}{M^{2}}\right)\right)^{-2},
\end{aligned}
$$

where, as usual, a prime marks the differentiation with respect to $\varphi$. The number of e-foldings during inflation is defined as

$$
N_{e} \equiv \int_{t_{i}}^{t_{f}} H d t=\int_{\varphi_{i}}^{\varphi_{f}} \frac{H}{\dot{\varphi}} d \varphi
$$

which in the slow-roll approximation can be written as

$$
N_{e}=-\int_{\varphi_{i}}^{\varphi_{f}} \frac{H\left(\Gamma+3 H\left(1+3 H^{2} / M^{2}\right)\right)}{V^{\prime}(\varphi)} d \varphi,
$$

where $\varphi_{f}\left(\varphi_{i}\right)$ is the value of the scalar field at the end (beginning) of the inflationary phase.

\section{Perturbations}

In this section we study scalar and tensor perturbations in our model. The scalar perturbations can be decomposed to entropy or isocurvature perturbations and adiabatic or curvature perturbations. In the framework of warm inflation, the scalar and radiation fields are interacting. Therefore, isocurvature perturbations are generated besides the adiabatic ones. This occurs because warm inflation can be considered as an inflationary model with two basic fields. Dissipative effects can produce a variety of spectral indexes, ranging between red and blue and thus producing the running blue to red spectral index suggested by WMAP data [23-25]. In the longitudinal gauge, the scalar metric perturbations of the FRW background are given by $[26,27]$

$$
d s^{2}=-(1+2 \Phi) d t^{2}+a^{2}(t)(1-2 \Psi) \delta_{i j} d x^{i} d x^{j}
$$

where $a(t)$ is the scale factor and $\Phi=\Phi(t, x)$ and $\Psi=\Psi(t, x)$ are the metric perturbations. The spatial dependence of perturbed quantities is in the form of plane waves $e^{i k \cdot x}$, where $k$ is the wave number. Therefore, the perturbation of the metric leads to the perturbation in the energy-momentum tensor 
through Einstein's field equations. The perturbed Einstein's field equations can be obtained as follows:

$$
\begin{gathered}
-3 H(H \Phi+\dot{\Psi})-\frac{k^{2}}{a^{2}} \\
=8 \pi G\left(\left(1+\frac{3 H^{2}}{M^{2}}\right) \dot{\varphi}^{2} \Phi+3 \frac{H^{2}}{M^{2}} \dot{\varphi} \Phi-\dot{\varphi} \delta \dot{\varphi}-V^{\prime} \delta \varphi\right) \\
\ddot{\Psi}+3 H(H \Phi+\dot{\Psi})+H \dot{\Phi}+2 \dot{H} \Phi+\frac{1}{3 a^{2}} k^{2}(\Phi-\Psi) \\
=8 \pi G\left(-\left(1+\frac{3 H^{2}}{M^{2}}\right) \dot{\varphi}^{2} \Phi+\frac{22}{M^{2}\left(4 \dot{H}+5 H^{2}\right) \dot{\varphi}} \Phi\right. \\
\left.+6 \frac{H}{M^{2}} \dot{\varphi}^{2} \Phi+\dot{\varphi} \delta \dot{\varphi}-V^{\prime} \delta \varphi\right) \\
H \Phi+\dot{\Psi}=8 \pi G\left(-\frac{4}{3 k} \rho_{R} a v+\left(1+3 \frac{H^{2}}{M^{2}}\right) \dot{\varphi} \delta \varphi\right) \\
\Psi-\Phi=\frac{M^{2}}{4 \pi G \dot{\varphi}^{2}} \Phi+\frac{M^{2}}{4 \pi G \dot{\varphi}^{2}} \Psi
\end{gathered}
$$

where $v$ appears from the decomposition of the velocity field as $\delta u_{i}=-\left(i a k_{j} / k\right) v e^{i k \cdot x}(j=1,2,3)[16]$ and we have omitted the subscript $k$. We perturb the scalar field equation of motion and the continuity equation for the radiation field as

$$
\begin{aligned}
& \left(1+3 \frac{H^{2}}{M^{2}}\right) \delta \ddot{\varphi}+\left(3 H\left(1+3 \frac{H^{2}}{M^{2}}+2 \frac{\dot{H}}{M^{2}}\right)+\Gamma\right) \delta \dot{\varphi} \\
& +\left(V^{\prime \prime}+\Gamma^{\prime} \dot{\varphi}\right) \delta \varphi+3\left(-1+9 \frac{H^{2}}{M^{2}}+\frac{2 \dot{H}}{M^{2}}+2 \frac{H}{M^{2}} \ddot{\varphi}\right) \dot{\Psi} \dot{\varphi} \\
& +3 \frac{H}{M^{2}} \dot{\varphi} \ddot{\Psi}-\left(1+9 \frac{H^{2}}{M^{2}}\right) \dot{\Phi} \dot{\varphi}=\left(-2 V^{\prime}+3 \Gamma \dot{\varphi}\right) \Phi, \\
& \delta \dot{\rho}_{R}+4 H \rho_{R}+\frac{4}{3} k a \rho_{R} v \\
& =4 \rho_{R} \dot{\Psi}+\dot{\varphi}^{2} \Gamma^{\prime} \delta \varphi+\Gamma \dot{\varphi}(2 \delta \dot{\varphi}-3 \dot{\varphi} \Phi) .
\end{aligned}
$$

We consider these equations in the slow-roll approximation and on large scales where $k \ll a H[28,29]$. In this situation, (26) become, respectively,

$$
\begin{gathered}
\left(3 H\left(1+3 \frac{H^{2}}{M^{2}}\right)+\Gamma\right) \delta \dot{\varphi}+\left(V^{\prime \prime}+\Gamma^{\prime} \dot{\varphi}\right) \delta \varphi \\
=\left(-2 V^{\prime}+3 \Gamma \dot{\varphi}\right) \Phi, \\
\frac{\delta \rho_{R}}{\rho_{R}} \simeq \frac{\Gamma^{\prime}}{\Gamma} \delta \varphi-3 \Phi,
\end{gathered}
$$

and (24) takes the following form:

$$
\Phi=8 \pi G \frac{\dot{\varphi}}{H}\left(1+3 \frac{H^{2}}{M^{2}}+\frac{\Gamma}{4 H}+\frac{\Gamma^{\prime} \dot{\varphi}}{48 H^{2}}\right) \delta \varphi,
$$

where we have used the relation of the velocity field as $v \simeq$ $-(k / 4 a H)\left(\Phi+\delta \rho_{R} / 4 \rho_{R}+\left(3 \Gamma \dot{\varphi} / 4 \rho_{R}\right) \delta \varphi\right)$.

We can solve the above set of equations by substituting (29) into (27), where we get

$$
\begin{aligned}
& \left(3 H\left(1+3 \frac{H^{2}}{M^{2}}\right)+\Gamma\right) \delta \dot{\varphi}+\left(V^{\prime \prime}+\Gamma^{\prime} \dot{\varphi}\right) \delta \varphi \\
& \simeq 8 \pi G \frac{\dot{\varphi}}{H}\left(-2 V^{\prime}+3 \Gamma \dot{\varphi}\right)\left(1+3 \frac{H^{2}}{M^{2}}+\frac{\Gamma}{4 H}+\frac{\Gamma^{\prime} \dot{\varphi}}{48 H^{2}}\right) \delta \varphi .
\end{aligned}
$$

Now, by defining a function $\chi$ as

$$
\chi=\frac{V \delta \varphi}{V^{\prime}} \exp \left(\int \frac{\Gamma^{\prime}}{\Gamma+3 H\left(1+3\left(H^{2} / M^{2}\right)\right)} d \varphi\right),
$$

(30) can be rewritten as

$$
\begin{aligned}
\frac{\chi^{\prime}}{\chi} \simeq & \frac{V^{\prime \prime}}{V^{\prime}}-\frac{\Gamma^{\prime}}{\left(\Gamma+3 H\left(1+3\left(H^{2} / M^{2}\right)\right)\right)} \\
& -\left(\frac{16 \pi G V^{\prime}}{H\left(\Gamma+3 H\left(1+3\left(H^{2} / M^{2}\right)\right)\right)}\right. \\
& \left.+\frac{24 \pi G \Gamma V^{\prime}}{H\left(\Gamma+3 H\left(1+3\left(H^{2} / M^{2}\right)\right)\right)^{2}}\right) \\
& \times\left(\left(1+3 \frac{H^{2}}{M^{2}}\right)+\frac{\Gamma}{4 H}\right. \\
& \left.\quad-\frac{\Gamma^{\prime} V^{\prime}}{48 H^{2}\left(\Gamma+3 H\left(1+3\left(H^{2} / M^{2}\right)\right)\right)}\right) .
\end{aligned}
$$

A solution of this equation is given as

$\chi(\varphi)$

$\simeq C$

$$
\begin{aligned}
\times \exp \left(\int \left\{\frac{V^{\prime \prime}}{V^{\prime}}-\frac{\Gamma^{\prime}}{\left(\Gamma+3 H\left(1+3\left(H^{2} / M^{2}\right)\right)\right)}\right.\right. \\
-\left(\frac{16 \pi G V^{\prime}}{H\left(\Gamma+3 H\left(1+3\left(H^{2} / M^{2}\right)\right)\right)}\right. \\
\left.\quad+\frac{24 \pi G \Gamma V^{\prime}}{H\left(\Gamma+3 H\left(1+3\left(H^{2} / M^{2}\right)\right)\right)^{2}}\right) \\
\quad\left(\left(1+3 \frac{H^{2}}{M^{2}}\right)+\frac{\Gamma}{4 H}\right. \\
\left.\left.\left.\quad-\frac{\Gamma^{\prime} V^{\prime}}{48 H^{2}\left(\Gamma+3 H\left(1+3\left(H^{2} / M^{2}\right)\right)\right)}\right)\right\} d \varphi\right),
\end{aligned}
$$


where $C$ is an integration constant. So, from (31), we find

$$
\begin{aligned}
& \delta \varphi \\
& \simeq \frac{C V^{\prime}}{V} \\
& \times \exp \left(\int \left\{\frac{V^{\prime \prime}}{V^{\prime}}-\frac{2 \Gamma^{\prime}}{\left(\Gamma+3 H\left(1+3\left(H^{2} / M^{2}\right)\right)\right)}\right.\right. \\
& -\left(\frac{16 \pi G V^{\prime}}{H\left(\Gamma+3 H\left(1+3\left(H^{2} / M^{2}\right)\right)\right)}\right. \\
& \left.+\frac{24 \pi G \Gamma V^{\prime}}{H\left(\Gamma+3 H\left(1+3\left(H^{2} / M^{2}\right)\right)\right)^{2}}\right) \\
& \times\left(\left(1+3 \frac{H^{2}}{M^{2}}\right)+\frac{\Gamma}{4 H}\right. \\
& \left.\left.\left.-\frac{\Gamma^{\prime} V^{\prime}}{48 H^{2}\left(\Gamma+3 H\left(1+3\left(H^{2} / M^{2}\right)\right)\right)}\right)\right\} d \varphi\right) .
\end{aligned}
$$

For simplicity we define the following quantity:

$$
\begin{gathered}
\mathbf{F} \equiv \int\left\{\frac{V^{\prime \prime}}{V^{\prime}}-\frac{2 \Gamma^{\prime}}{\left(\Gamma+3 H\left(1+3\left(H^{2} / M^{2}\right)\right)\right)}\right. \\
-\left(\frac{16 \pi G V^{\prime}}{H\left(\Gamma+3 H\left(1+3\left(H^{2} / M^{2}\right)\right)\right)}\right. \\
\left.\quad+\frac{24 \pi G \Gamma V^{\prime}}{H\left(\Gamma+3 H\left(1+3\left(H^{2} / M^{2}\right)\right)\right)^{2}}\right) \\
\times\left(\left(1+3 \frac{H^{2}}{M^{2}}\right)+\frac{\Gamma}{4 H}\right. \\
\left.\left.\quad-\frac{\Gamma^{\prime} V^{\prime}}{48 H^{2}\left(\Gamma+3 H\left(1+3\left(H^{2} / M^{2}\right)\right)\right)}\right)\right\} d \varphi .
\end{gathered}
$$

With this definition, we can rewrite (34) as

$$
\delta \varphi \simeq \frac{C V^{\prime}}{V} \exp (\mathbf{F}(\varphi))
$$

and, therefore, the density perturbation is given by

$$
\delta_{H}=\frac{4 \pi M_{p}^{2}}{5} \frac{V^{\prime}}{V} \exp (\mathbf{F}(\varphi)) \delta \varphi .
$$

The fluctuations of the scalar field are generated by thermal interaction with the radiation field, instead of quantum fluctuations

$$
(\delta \varphi)^{2} \simeq \frac{k_{F} T}{2 \pi^{2}}
$$

where the wave number $k_{F}$ is defined by $k_{F}=$ $\sqrt{\Gamma H+3 H^{2}\left(1+3\left(H^{2} / M^{2}\right)\right)}$ and corresponds to the freezeout scale at which dissipation damps out the thermally excited fluctuations. So, the density perturbation amplitude becomes

$$
\delta_{H}^{2}=\frac{8 M_{p}^{4}}{25} \frac{V^{\prime 2}}{V^{2}} \exp (2 \mathbf{F}(\varphi)) \sqrt{\Gamma H+3 H^{2}\left(1+3 \frac{H^{2}}{M^{2}}\right)} T .
$$

The scalar spectral index is given by

$$
n_{s}=1+\frac{d \ln \delta_{H}^{2}}{d \ln k}
$$

The interval in wave number is related to the number of efoldings by the relation

$$
d \ln k(\varphi)=-d N(\varphi)
$$

So we obtain

$$
\begin{aligned}
& n_{s}=1+\frac{4 V^{\prime \prime}}{H\left(\Gamma+3 H\left(1+3\left(H^{2} / M^{2}\right)\right)\right)} \\
& -\frac{V^{\prime 2}}{H V\left(\Gamma+3 H\left(1+3\left(H^{2} / M^{2}\right)\right)\right)} \\
& -\frac{\left(\Gamma^{\prime} H+H^{\prime} \Gamma+12 H^{\prime} H+36 H^{3} H^{\prime} / M^{2}\right) V^{\prime}}{H^{2}\left(\Gamma+3 H\left(1+3\left(H^{2} / M^{2}\right)\right)\right)^{2}} \\
& -\frac{2 \Gamma^{\prime} V^{\prime}}{H\left(\Gamma+3 H\left(1+3\left(H^{2} / M^{2}\right)\right)\right)^{2}} \\
& -\left(\frac{16 \pi G V^{\prime}}{H\left(\Gamma+3 H\left(1+3\left(H^{2} / M^{2}\right)\right)\right)}\right. \\
& \left.+\frac{24 \pi G \Gamma V^{\prime}}{H\left(\Gamma+3 H\left(1+3\left(H^{2} / M^{2}\right)\right)\right)^{2}}\right) \\
& \times \frac{V^{\prime}}{H\left(\Gamma+3 H\left(1+3\left(H^{2} / M^{2}\right)\right)\right)} \\
& \times\left(\left(1+3 \frac{H^{2}}{M^{2}}\right)+\frac{\Gamma}{4 H}\right. \\
& \left.-\frac{\Gamma^{\prime} V^{\prime}}{48 H^{2}\left(\Gamma+3 H\left(1+3\left(H^{2} / M^{2}\right)\right)\right)}\right) \text {. }
\end{aligned}
$$

The running of the spectral index which is defined as

$$
\alpha_{s}=\frac{d n_{s}}{d \ln k}
$$


in our model takes the following form:

$$
\begin{aligned}
& \alpha_{s}=\frac{\left(2 \mathbf{F}^{\prime \prime} V^{\prime}+2 \mathbf{F}^{\prime} V^{\prime \prime}\right) V^{\prime}}{H^{2}\left(\Gamma+3 H\left(1+3\left(H^{2} / M^{2}\right)\right)\right)^{2}} \\
& -\left(\mathbf{F}^{\prime} V^{\prime 2}-V^{\prime \prime} V^{\prime}\right)\left(36 \frac{H^{3} H^{\prime}}{M^{2}}+\Gamma^{\prime} H\right. \\
& \left.+H^{\prime} \Gamma+3 H H^{\prime}\right) \\
& \times\left(H^{3}\left(\Gamma+3 H\left(1+3 \frac{H^{2}}{M^{2}}\right)\right)^{3}\right)^{-1} \\
& -\frac{2 V^{\prime \prime}}{H V\left(\Gamma+3 H\left(1+3\left(H^{2} / M^{2}\right)\right)\right)} \\
& -\left(\Gamma^{\prime} H V+\Gamma H^{\prime} V+\Gamma H V^{\prime}+6 H H^{\prime} V\right. \\
& \left.+3 H^{2} V^{\prime}+\frac{36 H^{3} H^{\prime} V}{M^{2}+9 H^{4} / M^{2}} V^{\prime}\right) 2 V^{\prime} \\
& \times\left(H^{2} V^{2}\left(\Gamma+3 H\left(1+3 \frac{H^{2}}{M^{2}}\right)\right)^{2}\right)^{-1} \\
& +\frac{4 V^{\prime} V^{\prime \prime \prime}}{H^{2}\left(\Gamma+3 H\left(1+3 H^{2} / M^{2}\right)\right)^{2}} \\
& +\frac{2 V^{\prime}\left(36\left(H^{3} / M^{2}\right) H^{\prime}+6 H H^{\prime}+\Gamma^{\prime} H+H^{\prime} \Gamma\right)}{H^{2}\left(\Gamma+3 H\left(1+3 H^{2} / M^{2}\right)\right)^{2}} \\
& \times\left[\frac{4 V^{\prime \prime}}{H\left(\Gamma+3 H\left(1+3 H^{2} / M^{2}\right)\right)}+2 \eta\right] \\
& -12 M_{p}^{2} \varepsilon\left(6 H H^{\prime \prime}+6 H^{\prime 2}+108 H^{2} H^{\prime 2}\right. \\
& \left.+36 \frac{H^{3}}{M^{2}} H^{\prime \prime}+\Gamma^{\prime \prime} H+2 H^{\prime} \Gamma^{\prime}+H^{\prime \prime} \Gamma\right) \\
& \times\left(\Gamma+3 H\left(1+\frac{3 H^{2}}{M^{2}}\right)\right)^{-2}
\end{aligned}
$$

Now we pay attention to the tensorial perturbations. As has been mentioned in [30], the generation of tensor perturbations during inflation period produces stimulated emission in the thermal background of gravitational waves. This process changes the power spectrum of the tensor modes by an extra, temperature-dependent factor given by $\operatorname{coth}(k / 2 T)$. Therefore, the spectrum of tensor perturbations is given by

$$
A_{T}^{2}=\left.\frac{4}{25 \pi M_{p}^{2}} H^{2} \operatorname{coth}\left(\frac{k}{2 T}\right)\right|_{k=a H} .
$$

The ratio between the amplitudes of tensor and scalar perturbations (tensor-to-scalar ratio) is given by

$$
\begin{aligned}
r & =\left(\frac{A_{T}^{2}}{P_{R}}\right)_{k=a H} \\
& \simeq \frac{4 H^{2} V^{2} \exp (-2 \mathbf{F})}{25 M_{p}^{2} V^{\prime 2} \sqrt{\Gamma+3 H\left(1+3\left(H^{2} / M^{2}\right)\right)} T} \operatorname{coth}\left(\frac{k}{2 T}\right),
\end{aligned}
$$

where $P_{R}=(25 / 4) \delta_{H}^{2}$

This is the complete treatment of perturbations in this setup. In what follows, we consider two specific inflaton potentials to study the inflationary aspects of this model and confrontation with recent observational data from Planck + WMAP9 + $\mathrm{BAO}+\mathrm{H}_{0}[23-25]$.

\section{Specific Examples}

4.1. Chaotic Inflation. With $V(\varphi)=(1 / 2) m^{2} \varphi^{2}$, the number of e-foldings in the slow-roll approximation at the end of the inflation is given by

$$
N=\left[\frac{x^{2}}{2}(1+Q)+\frac{\alpha x^{4}}{8}\right]-\left[\frac{x_{f}^{2}}{2}(1+Q)+\frac{\alpha x_{f}^{4}}{8}\right]
$$

where $x, \alpha$, and $Q$ are defined as follows:

$$
x=\frac{\varphi}{M_{p}}, \quad \alpha=\frac{m^{2}}{M^{2}}, \quad Q=\frac{\Gamma}{3 H} .
$$

Note that if we set $\varepsilon=1$ for the end of the inflation, the dimensionless field at the end of the inflation attains the following form:

$$
x_{f}^{2}=\frac{-1}{2}+\sqrt{\frac{1}{4}+\frac{\alpha}{(1+Q)^{2}}} .
$$

Substituting (49) into (47), we find $x^{2}$ as follows:

$$
\begin{aligned}
x^{2}=\frac{1}{2 \alpha(1+Q)}\left\{\begin{array}{l}
- \\
+(1+Q)^{2}
\end{array}\right. \\
+\left[16\left(\left(Q^{2}+1\right)^{2}+4 Q\left(Q^{2}+Q+1\right)\right)\right. \\
-32 \alpha+2 \alpha^{2} Q^{2}-96 \alpha Q^{2}+32 \alpha N
\end{aligned}
$$




$$
\left.\begin{array}{l}
+32 \alpha \sqrt{(Q+1)^{2}+4 \alpha} \\
-96 \alpha Q+64 \alpha N Q+32 \alpha N Q^{2} \\
-4 \alpha^{2} \sqrt{1+\frac{4 \alpha}{(1+Q)^{2}} Q} \\
-2 \alpha^{2} \sqrt{1+\frac{4 \alpha}{(1+Q)^{2}} Q^{2}} \\
-32 \alpha Q^{3}+64 \alpha \sqrt{1+2 Q}+4 \alpha Q \\
+32 \alpha \sqrt{(1+Q)^{2}+4 \alpha Q}+4 \alpha^{2} Q \\
\left.+4 \alpha^{3}-2 \alpha^{2} \sqrt{1+\frac{4 \alpha}{(1+Q)^{2}}}\right]^{1 / 2}
\end{array}\right\} .
$$

Using (42) and (46), the scalar spectral index and the tensor-to-scalar ratio in the warm chaotic inflation are given by

$$
\begin{aligned}
n_{s}= & 1+\frac{4}{x^{2}\left(Q+1+\alpha x^{2} / 2\right)}-\frac{\left(8 \alpha x^{2}+16\right)}{x^{2}\left(Q+1+\alpha x^{2} / 2\right)^{2}} \\
& -\left(\frac{8}{x^{2}\left(Q+1+\alpha x^{2} / 2\right)^{2}}+\frac{12 Q}{x^{2}\left(Q+1+\alpha x^{2} / 2\right)^{3}}\right) \\
& \times\left(1+\frac{\alpha x^{2}}{2}+\frac{3}{4} Q-\frac{Q}{4 x^{2}\left(Q+1+\alpha x^{2} / 2\right)}\right), \\
r= & \frac{\sqrt{2} x^{3} \exp (2 \mathbf{F})}{\sqrt{Q+\left(1+\alpha x^{2} / 2\right)} T} \operatorname{coth}\left(\frac{k}{2 T}\right)
\end{aligned}
$$

respectively, where $x$ is given by the relation (50). The left panel of Figure 1 shows the behavior of the tensor-to-scalar ratio versus the scalar spectral index with $\alpha=1000$. For $N=50$, the model with $4<Q<53.5$, for $N=60$, the model with $3.94<Q<52.73$, and for $N=70$, the model with $3.90<$ $Q<51.86$ lie inside the $95 \%$ CL of the Planck + WMAP9 $+\mathrm{BAO}+\mathrm{H}_{0}$ joint data. We have also plotted the evolution of the running of the scalar spectral index versus the scalar spectral index in the right panel of Figure 1. For this case, the running is negative and in some range of $\alpha$ compatible with recent observational data. From another perspective, we can fix $Q$ at, for instance, $Q=1000$ and let $\alpha$ vary in order to see the effect of nonminimal derivative coupling. In Figure 2, we survey the effects of the nonminimal derivative decoupling. The left panel of this figure shows the behavior of the tensorto-scalar ratio versus the scalar spectral index with $Q=1000$. For $N=50$, the model with $23<\alpha<141.3$, for $N=60$, the model with $13.67<\alpha<140.05$, and for $N=70$, the model with $16<\alpha<127.83$ are compatible with observational data. Also, evolution of the running of the scalar spectral index versus the scalar spectral index in the background of Planck + WMAP9 $+\mathrm{BAO}+\mathrm{H}_{0}$ joint data is shown in the right panel of Figure 2. For this case, the running is negative in some range of $\alpha$ and lies inside the 95\% CL of the Planck + WMAP9 + $\mathrm{BAO}+H_{0}$.

4.2. Natural Inflation. Natural inflation is characterized by the potential

$$
V(\varphi)=\Lambda^{4}\left[1+\cos \left(\frac{\varphi}{f}\right)\right]
$$

where $\Lambda$ and $f$ are constants having the dimension of mass. The number of e-foldings at the end of inflation with the above potential is given by

$$
\begin{aligned}
N=\left[\ln \left(\frac{1}{\sin \chi}-\frac{1}{\tan \chi}\right)(Q+1)\right. \\
+2 \gamma\left(\ln \frac{1}{\sin \chi}-\frac{1}{\tan \chi}+\ln \sin \chi\right) \\
+\ln (\sin \chi)(Q+1)+\gamma \cos \chi] \\
-\left[\ln \left(\frac{1}{\sin \chi_{f}}-\frac{1}{\tan \chi f}\right)(Q+1)\right. \\
\quad+2 \gamma\left(\ln \frac{1}{\sin \chi_{f}}-\frac{1}{\tan \chi_{f}}+\ln \sin \chi_{f}\right) \\
\left.\quad+\ln \left(\sin \chi_{f}\right)(r+1)+\gamma \cos \chi_{f}\right],
\end{aligned}
$$

where

$$
\chi=\frac{\varphi}{f}, \quad \chi_{f}=\frac{\varphi_{f}}{f}, \quad \gamma=\frac{f^{2} \Lambda^{4}}{M^{2} M_{p}^{4}} .
$$

So, from (16) the value of $\chi$ at the end of inflation takes the following form:

$$
\cos \chi_{f}=-1+\frac{\sqrt{4 Q^{2}+12 Q+16 \gamma+9}-3-2 Q}{4 \gamma} .
$$




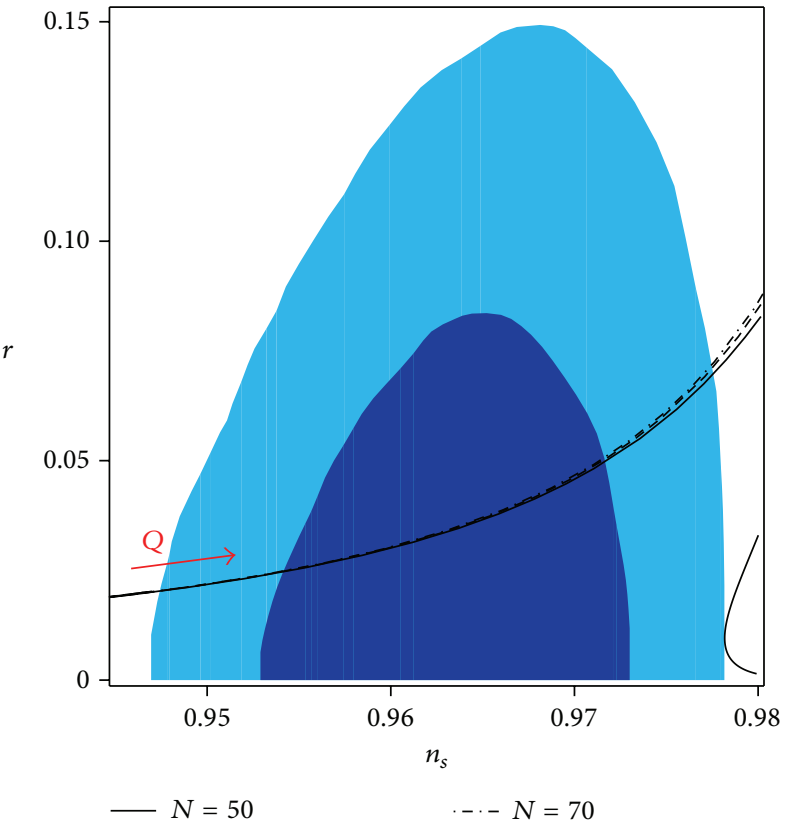

(a)

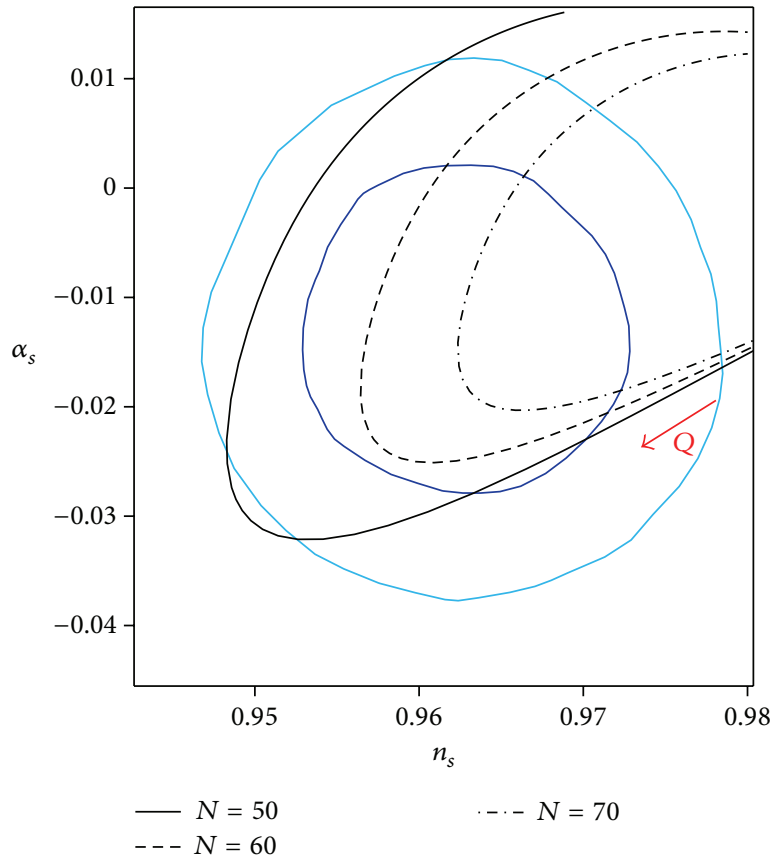

(b)

Figure 1: Evolution of the tensor-to-scalar ratio (a) and running of the scalar spectral index (b) versus the scalar spectral index for chaotic potential in the background of Planck + WMAP9 $+\mathrm{BAO}+H_{0}$ data. The figure has been plotted for $N=50,60$, and 70 and with $\alpha=1000$ with varying $Q$.

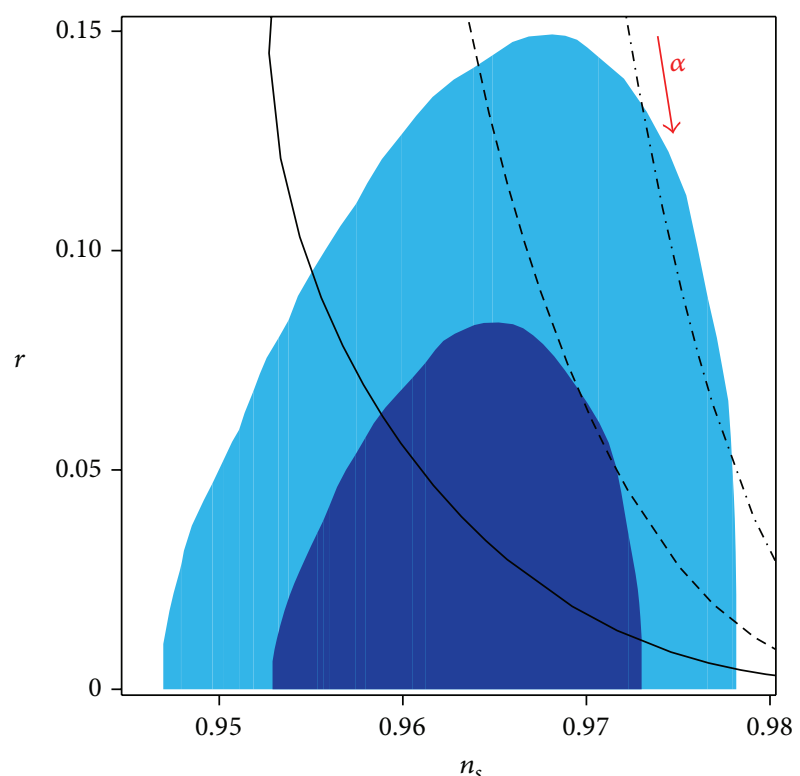

$\begin{aligned}-N & =50 \\ --N & =60\end{aligned}$

(a)

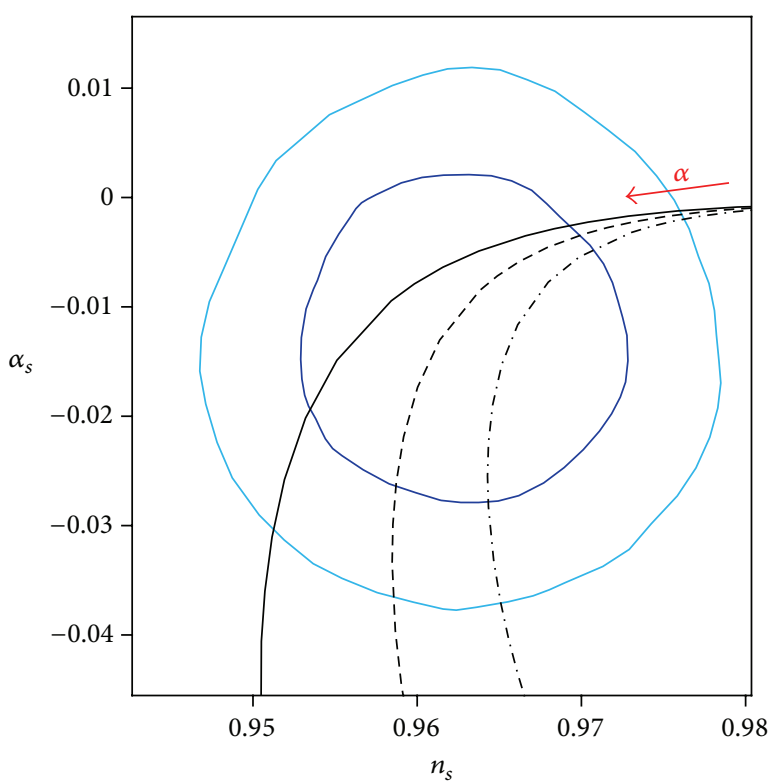

$\begin{aligned} N & =50 \\ --N & =60\end{aligned}$

(b)

FIGURE 2: Evolution of the tensor-to-scalar ratio (a) and running of the scalar spectral index (b) versus the scalar spectral index for chaotic potential in the background of Planck + WMAP9 $+\mathrm{BAO}+H_{0}$ data. The figure has been plotted for $N=50,60$, and 70 and with $Q=1000$ and varying $\alpha$. 
Substituting (55) into (53), $\chi$ is determined fully for given values of $\gamma, N$, and $Q$. Then $n_{s}$ and $r$ can be obtained, respectively, as follows:

$$
\begin{aligned}
n_{s}= & -\frac{\cos \chi}{(1+\cos \chi)(Q+1+\gamma(1+\cos \chi))} \\
& -\frac{\sin \chi Q+\sin \chi+\gamma \sin \chi(1+\cos \chi)-\sin ^{2} \chi}{(1+\cos \chi)^{2}(Q+1+\gamma(1+\cos \chi))^{2}} \\
& -\frac{6 \sin ^{2} \chi}{(1+\cos \chi)^{2}(Q+1+\gamma(1+\cos \chi))} \\
& -\frac{(1+\cos \chi)^{2}(Q+1+\gamma(1+\cos \chi))^{2}}{2 \sin ^{2} \chi Q} \\
& -\left(\frac{6 \sin ^{2} \chi}{9(1+\cos \chi)^{2}(Q+1+\gamma(1+\cos \chi))^{2}}\right. \\
& \left.+\frac{(1+\cos \chi)^{3 / 2} \exp ^{2} \chi Q}{3(1+\cos \chi)^{2}(Q+1+\gamma(1+\cos \chi))^{3}}\right) \\
& +\frac{6 Q \sin ^{2} \chi}{\left(Q+1+\gamma\left(1+\cos ^{2} \chi\right)\right)} \\
& \left.+\frac{k}{2 T}\right)
\end{aligned}
$$

Now we consider the observational constraints on natural inflation parameters in our setup. As in the previous example, we consider three values of the number of e-folds. In Figure 3(a), we have shown evolution of the tensor-to-scalar ratio versus the scalar spectral index with $\gamma=100$ and variable $Q$. For $N=50$, the model with $Q>176.55$, for $N=60$, the model with $Q>177.122$, and for $N=70$, the model with $Q>177.05$ are compatible with Planck + WMAP9 + BAO $+H_{0}$ data. The right panel of Figure 3 shows the behavior of the running of the spectral index versus the scalar spectral index in the background of the Planck + WMAP9 + BAO $+H_{0}$ joint data. The Figure has been plotted with $\gamma=100$. In our setup with natural inflation potential, the running of the scalar spectral index is negative and lies inside of the 95\% CL of the Planck + WMAP9 $+\mathrm{BAO}+H_{0}$ joint data. Now we consider the opposite case; we fix $Q$ and let $\gamma$ to be variable. We fix $Q$ at $Q=100$. The left panel of Figure 4 shows the behavior of the tensor-to-scalar ratio versus the scalar spectral index with $Q=100$ and variable $\gamma$. For $N=50$, the model with $156<\gamma<273.6$, for $N=60$, the model with $156.03<\gamma<273.04$, and for $N=70$, the model with $156.08<\gamma<273.31$ are compatible with data. The right panel of Figure 4 shows evolution of the running of the spectral index versus the scalar spectral index. For $N=50$, the model with $112.37<\gamma<211.71$, for $N=60$, the model with $113.5<\gamma<213.38$, and for $N=70$, the model with $114.24<\gamma<216.88$ lie inside the 95\% CL of the Planck + WMAP9 $+\mathrm{BAO}+\mathrm{H}_{0}$ joint data.

In Tables 1 and 2, we have summarized the results of numerical analysis for different values of $N$ for the above mentioned potentials in our warm inflation model with nonminimal derivative coupling. We have specified the range of $\alpha, \gamma$, and $Q$ parameters in which the model is compatible with Planck + WMAP9 $+\mathrm{BAO}+H_{0}$ joint data.

\section{Non-Gaussianity}

In this section we study non-Gaussianity of the primordial perturbations in our model. We calculate the three-point correlation function (bispectrum) of the density perturbation distribution in the Fourier space. Inflaton field equation of motion is given by the following expression:

$$
\dot{\varphi}=-\frac{V^{\prime}(\varphi)}{3 H\left(Q+\left(1+\left(3 H^{2} / M^{2}\right)\right)\right)} .
$$

In order to study the fluctuations of the inflaton field $\delta \varphi(x, t)$, it is assumed that the fluctuations are small and the full inflaton field is emerged as $\varphi(x, t)=\varphi_{0}(t)+\delta \varphi(x, t)$, where $\varphi_{0}$ is homogeneous background field and $\delta \varphi(x, t) \ll$ $\varphi_{0}(t)$. Therefore, the full inflaton equation of motion with fluctuation in our setup will be as follows:

$$
\begin{aligned}
\frac{d \varphi(x, t)}{d t}= & \frac{1}{3 H\left(Q+\left(1+\left(3 H^{2} / M^{2}\right)\right)\right)} \\
& \times\left[e^{-2 H t} \nabla^{2} \varphi(x, t)-V^{\prime}(\varphi(x, t))+\eta(x, t)\right],
\end{aligned}
$$

where $\eta(x, t)$ is a Gaussian noise term. The fluctuationdissipation theorem determines the properties of the noise, as shown in $[31,32]$. So, this term in the momentum space has the following properties:

$$
\begin{gathered}
\langle\eta\rangle=0, \\
\left\langle\eta(k, t) \eta\left(k^{\prime}, t^{\prime}\right)\right\rangle=2 \Gamma T(2 \pi)^{3} \delta^{3}\left(k-k^{\prime}\right) \delta\left(t-t^{\prime}\right) .
\end{gathered}
$$

In order to calculate the bispectrum, we consider the inflaton field fluctuation up to the second order $\delta \varphi(x, t)=\delta \varphi_{1}(x, t)+$ $\delta \varphi_{2}(x, t)$, where $\delta \varphi_{1}$ is the first order of $\delta \varphi$ and $\delta \varphi_{2}$ is the second order. Thus, the equations of motion of the first 


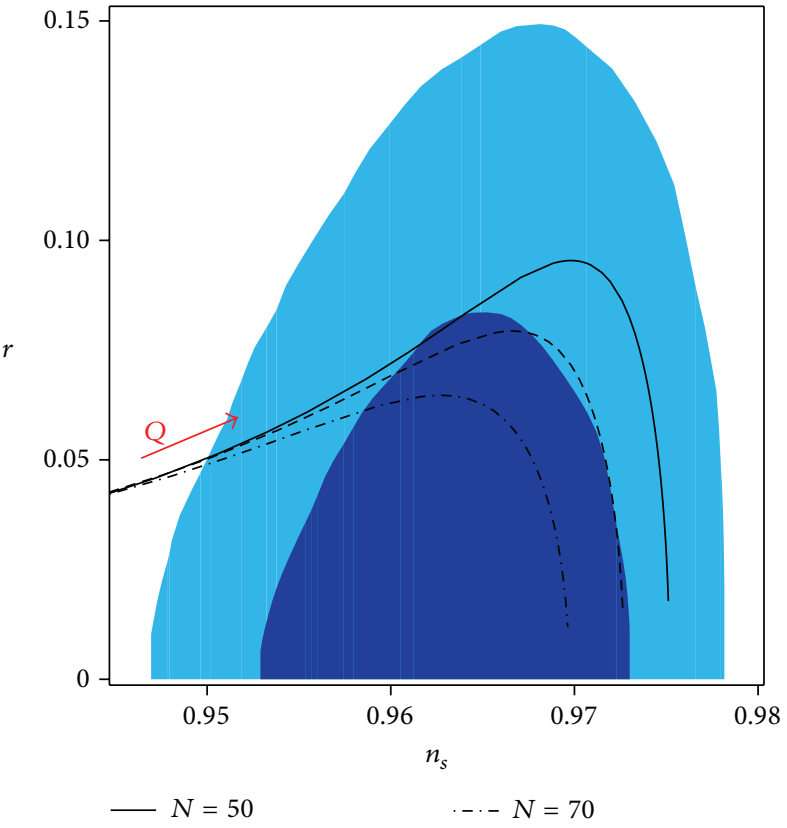

(a)

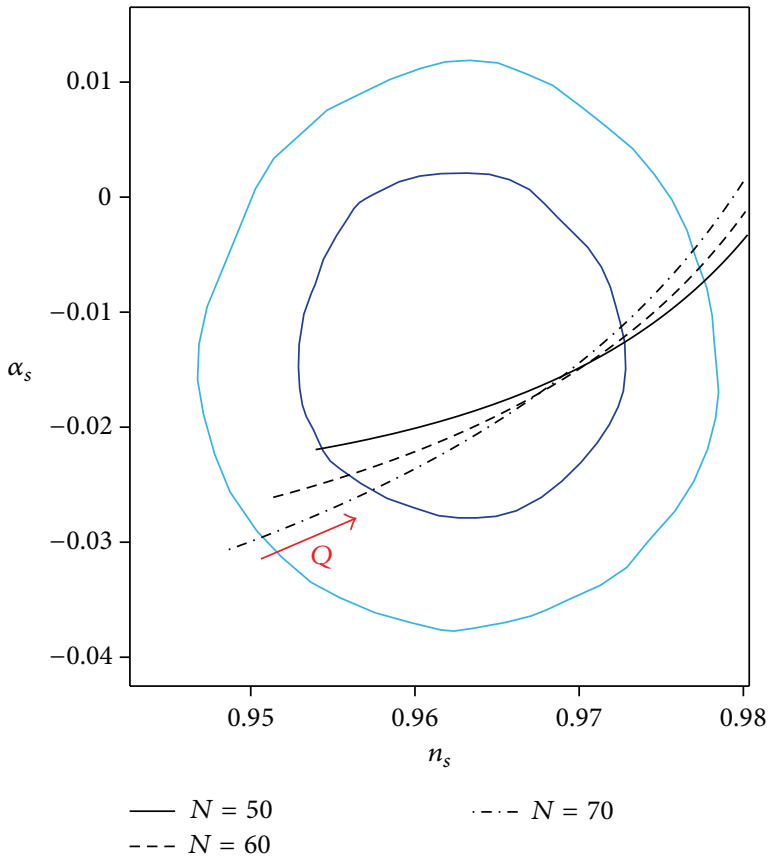

(b)

Figure 3: Evolution of the tensor-to-scalar ratio (a) and running of the scalar spectral index (b) versus the scalar spectral index for natural inflation in the background of Planck + WMAP9 $+\mathrm{BAO}+\mathrm{H}_{0}$ data. The figure has been plotted for $N=50,60$, and 70, with $\gamma=100$ and $Q$ as a free parameter.

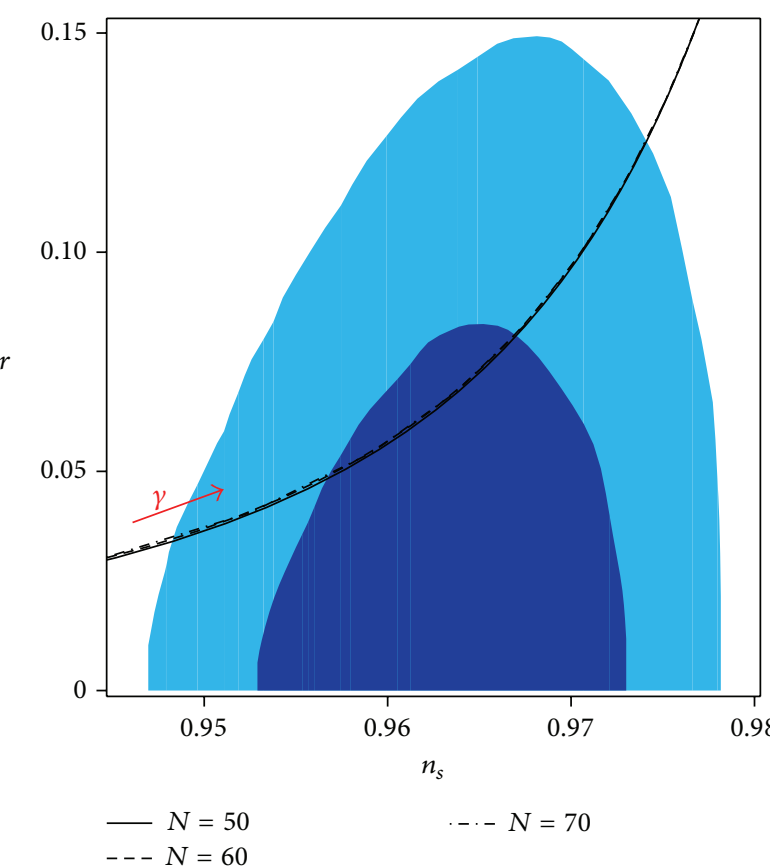

(a)

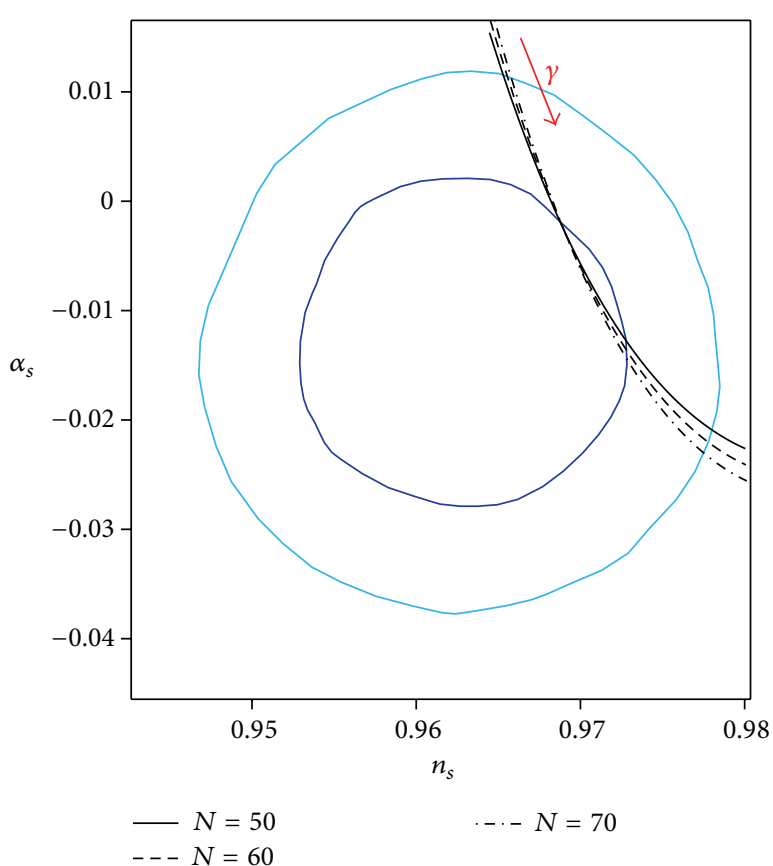

(b)

FiguRE 4: Evolution of the tensor-to-scalar ratio (a) and running of the scalar spectral index (b) versus the scalar spectral index for natural inflation in the background of Planck + WMAP9 $+\mathrm{BAO}+H_{0}$ data. The figure has been plotted for $N=50,60$, and 70 , with $Q=100$ and $\gamma$ as a free parameter. 
TABLE 1: Constraints on $\alpha=m^{2} / M^{2}$ for chaotic potential with $Q=1000$ and on $\gamma=\left(f^{2} \Lambda^{4}\right) /\left(M^{2} M_{p}^{4}\right)$ for natural potential with $Q=100$ for which the model of warm inflation with nonminimal derivative coupling is compatible with the Planck $+\mathrm{WMAP} 9+\mathrm{BAO}+H_{0}$ joint data.

\begin{tabular}{lcccc}
\hline Potential & Probe & $N=50$ & $N=60$ & $N=70$ \\
\hline$\frac{1}{2} m^{2} \varphi^{2}$ & $r-n_{s}$ & $23<\alpha<141.3$ & $13.67<\alpha<140.05$ & $16<\alpha<127.83$ \\
& $\alpha_{s}-n_{s}$ & $2.12<\alpha<41.28$ & $2.10<\alpha<43.7$ & $2.09<\alpha<45.36$ \\
\hline$\Lambda^{4}\left[1+\cos \left(\frac{\varphi}{f}\right)\right]$ & $r-n_{s}$ & $156<\gamma<273.6$ & $156.03<\gamma<273.04$ & $156.08<\gamma<273.31$ \\
& $\alpha_{s}-n_{s}$ & $112.37<\gamma<211.71$ & $113.5<\gamma<213.38$ & $114.24<\gamma<216.88$ \\
\hline
\end{tabular}

TABLE 2: Constraints on $Q$ with $\alpha=1000$ and $\gamma=100$ for which the model of warm inflation with nonminimal derivative coupling is compatible with the Planck + WMAP9 $+\mathrm{BAO}+H_{0}$ joint data.

\begin{tabular}{lcccc}
\hline Potential & Probe & $N=50$ & $N=60$ & $N=70$ \\
\hline$\frac{1}{2} m^{2} \varphi^{2}$ & $r-n_{s}$ & $4<Q<53.5$ & $3.94<Q<52.73$ & $3.90<Q<51.86$ \\
$\Lambda^{4}\left[1+\cos \left(\frac{\varphi}{f}\right)\right]$ & $\alpha_{s}-n_{s}$ & $12<Q<893$ & $12.23<Q<803$ & $12.31<Q<724$ \\
\hline
\end{tabular}

and second order fluctuations in a time period $t_{n}-t_{n-1}=1 / H$ are

$$
\begin{aligned}
\frac{d}{d t}\left(\delta \varphi_{1}(k, t)\right)= & \frac{1}{3 H\left(Q+\left(1+3 H^{2} / M^{2}\right)\right)} \\
& \times\left[-k^{2} \delta \varphi_{1}(k, t)-V^{\prime \prime}\left(\varphi_{0}(t)\right) \delta \varphi_{1}(k, t)\right. \\
& +\eta(k, t)], \\
\frac{d}{d t}\left(\delta \varphi_{2}(k, t)\right)= & \frac{1}{3 H\left(Q+\left(1+3 H^{2} / M^{2}\right)\right)} \\
& \times\left[-k^{2} \delta \varphi_{2}(k, t)-V^{\prime \prime}\left(\varphi_{0}(t)\right) \delta \varphi_{2}(k, t)\right. \\
& \left.\quad-\frac{1}{2} V^{\prime \prime \prime}\left(\varphi_{0}(t)\right) \delta \varphi_{1}^{2}(k, t)\right] .
\end{aligned}
$$

Therefore, the solution of the above equations will be as follows:

$$
\begin{aligned}
\delta \varphi_{1}(k, t)= & A\left(k, t-t_{n-1}\right) \int_{t_{n-1}}^{t} d t^{\prime} \frac{\eta\left(k, t^{\prime}\right)}{3 H\left(Q+\left(1+\left(3 H^{2} / M^{2}\right)\right)\right)} \\
& \times A^{-1}\left(k, t^{\prime}-t_{n-1}\right) \\
& +A\left(k, t-t_{n-1}\right) \delta \varphi_{1}\left(k e^{-H\left(t_{n}-t_{n-1}\right)}, t_{n-1}\right), \\
\delta \varphi_{2}(k, t)= & A\left(k, t-t_{n-1}\right) \\
& \times \int_{t_{n-1}}^{t} d t^{\prime} B\left(t^{\prime}\right) \\
& \times\left[\int \frac{d p^{3}}{(2 \pi)^{3}} \delta \varphi_{1}\left(p, t^{\prime}\right) \delta \varphi_{1}\left(k-p, t^{\prime}\right)\right]
\end{aligned}
$$

$$
\begin{gathered}
\times A^{-1}\left(k, t^{\prime}-t n-1\right) \\
+A\left(k, t-t_{n-1}\right) \delta \varphi_{1}\left(k e^{-H\left(t_{n}-t_{n-1}\right)}, t_{n-1}\right),
\end{gathered}
$$

where $A$ and $B$ are defined as follows:

$$
\begin{gathered}
A(k, t)=\exp \left[-\int_{t_{0}}^{t}\left(\frac{k^{2}}{3 H\left(Q+\left(1+3 H^{2} / M^{2}\right)\right)}\right.\right. \\
\left.\left.+\frac{V^{\prime \prime}\left(\varphi_{0}\left(t^{\prime}\right)\right)}{3 H\left(Q+\left(1+3 H^{2} / M^{2}\right)\right)}\right) d t^{\prime}\right] \\
B(t)=-\frac{V^{\prime \prime \prime}\left(\varphi_{0}(t)\right)}{3 H\left(Q+\left(1+3 H^{2} / M^{2}\right)\right)} .
\end{gathered}
$$

The second term on the right in both parts of (61) is memory terms. These memory terms within the Hubble time give the concept of freeze-out [33]. By definition of freeze-out, for $k \geq k_{F}$ this term damps away and for $k \leq k_{F}$ the memory term reminds within a Hubble time. By definition, $k_{F}$ can be obtained via the following condition:

$$
\frac{k_{F}^{2}+V^{\prime \prime}\left(\varphi_{0}\right)}{\Gamma H+3 H^{2}\left(1+3\left(H^{2} / M^{2}\right)\right)}=1 \text {. }
$$

For warm inflation $V^{\prime \prime}\left(\varphi_{0}\right)<\Gamma H$, so the freeze-out condition in this model can be simplified to

$$
k_{F}=\sqrt{\Gamma H+3 H^{2}\left(1+3 \frac{H^{2}}{M^{2}}\right)} .
$$

Note that the second term in square root comes from the nonminimal derivative coupling. Now, we obtain the bispectrum before the end of inflation. The three-point correlation 
function (when the number of e-folding is equal to 60) becomes

$$
\begin{aligned}
& \left\langle\delta \varphi\left(k_{1}, t\right) \delta \varphi\left(k_{2}, t\right) \delta \varphi\left(k_{3}, t\right)\right\rangle \\
& =A\left(k_{3}, t-t_{60}-\frac{1}{H}\right) \\
& \quad \times \int_{t_{60}-1 / H}^{t_{60}} A^{-1}\left(k_{3}, t^{\prime}-t_{60}-\frac{1}{H}\right) B\left(t^{\prime}\right) \\
& \quad \times\left[\int \frac{d^{3} p}{(2 \pi)^{3}}\left\langle\delta \varphi_{1}\left(k_{1}, t_{1}\right) \delta \varphi_{1}\left(p, t^{\prime}\right)\right\rangle\right. \\
& \left.\quad \times\left\langle\delta \varphi_{1}\left(k_{2}, t_{2}\right) \delta \varphi_{1}\left(k_{3}-p, t^{\prime}\right)\right\rangle\right] \\
& +A\left(k_{3}, t-t_{60}-\frac{1}{H}\right) \\
& \quad \times\left\langle\delta \varphi_{1}\left(k_{1}, t_{60}\right) \delta \varphi_{1}\left(k_{2}, t_{60}\right) \delta \varphi_{2}\left(k_{3} e^{-1}, t_{60}-\frac{1}{H}\right)\right\rangle \\
& +\left(k_{1} \longleftrightarrow k_{3}\right)+\left(k_{2} \longleftrightarrow k_{3}\right) .
\end{aligned}
$$

On the scales with $k<k_{f}$, we can take $A$ about unity. So we have

$$
\begin{aligned}
& \left\langle\delta \varphi\left(k_{1}, t\right) \delta \varphi\left(k_{2}, t\right) \delta \varphi\left(k_{3}, t\right)\right\rangle \\
& \approx B\left(t_{60}\right) \Delta t_{F} \\
& \quad \times\left[\int \frac{d^{3} p}{(2 \pi)^{3}}\left\langle\delta \varphi_{1}\left(k_{1}, t_{1}\right) \delta \varphi_{1}\left(p, t^{\prime}\right)\right\rangle\right. \\
& \quad \times\left\langle\delta \varphi_{1}\left(k_{2}, t_{2}\right) \delta \varphi_{1}\left(k_{3}-p, t^{\prime}\right)\right\rangle \\
& \left.+\left(k_{1} \longleftrightarrow k_{3}\right)+\left(k_{2} \longleftrightarrow k_{3}\right)\right]
\end{aligned}
$$

where

$$
\Delta t_{f} \equiv t_{H}-t_{F} \approx \frac{1}{H} \ln \left(\frac{k_{F}}{H}\right)
$$

where $t_{H}$ represents the time at Hubble crossing of the smallest of the three inflation perturbation modes and $t_{F}$ represents the time when the last of the three wave vectors thermalizes. The bispectrum for slow-roll, single field, supercooled inflation models, and also for the set of warm inflation models is given by $[31,32]$

$$
\begin{aligned}
& \left\langle\Phi\left(k_{1}\right) \Phi\left(k_{2}\right) \Phi\left(k_{3}\right)\right\rangle \\
& \quad=A_{\mathrm{inf}}(2 \pi)^{3} \delta^{3}\left(k_{1}+k_{2}+k_{3}\right)\left[P_{\Phi\left(k_{1}\right)} P_{\Phi\left(k_{2}\right)}+\text { perms }\right] .
\end{aligned}
$$

In our setup, the relation between the scalar field fluctuation and the gravitational field is defined by

$$
\begin{aligned}
\Phi(k)=-3 \frac{H}{\dot{\varphi}}( & \frac{\left(1+3\left(H^{2} / M^{2}\right)\right)}{\left(Q+\left(1+3\left(H^{2} / M^{2}\right)\right)\right)} \\
& +\frac{\Gamma}{4 H\left(Q+\left(1+3\left(H^{2} / M^{2}\right)\right)\right)} \\
& \left.-\frac{V^{\prime} \Gamma^{\prime}}{48 H^{3}\left(Q+\left(1+3\left(H^{2} / M^{2}\right)\right)\right)^{2}}\right) \delta \varphi_{k} .
\end{aligned}
$$

Therefore, $A_{\text {inf }}$ in the high-dissipation regime of warm inflation with nonminimal derivative coupling is given by

$$
\begin{aligned}
A_{\text {inf }}=-\frac{1}{3}\left(\frac{\dot{\varphi}}{H}\right)( & \frac{\left(1+3\left(H^{2} / M^{2}\right)\right)}{\left(Q+\left(1+3\left(H^{2} / M^{2}\right)\right)\right)} \\
& +\frac{\Gamma}{4 H\left(Q+\left(1+3\left(H^{2} / M^{2}\right)\right)\right)} \\
& \left.-\frac{V^{\prime} \Gamma^{\prime}}{48 H^{3}\left(Q+\left(1+3\left(H^{2} / M^{2}\right)\right)\right)^{2}}\right)^{-1} \\
& \times\left(\frac{1}{H} \ln \left(\frac{k_{F}}{H}\right)\left(\frac{V^{\prime \prime \prime}\left(\varphi_{0}\left(t_{F}\right)\right)}{3 H\left(Q+\left(1+3\left(H^{2} / M^{2}\right)\right)\right)}\right)\right) .
\end{aligned}
$$

The $A_{\text {inf }}$ parameter for the pure warm inflation (without the nonminimal derivative coupling) is obtained as [19]

$$
A_{\mathrm{inf}}=-\frac{10}{3}\left(\frac{\dot{\varphi}}{H}\right)\left[\frac{1}{H} \ln \left(\frac{k_{F}}{H}\right)\left(\frac{V^{\prime \prime \prime}\left(\varphi_{0}\left(t_{F}\right)\right)}{3 H Q}\right)\right] .
$$

We see that the non-Gaussianity in the high-dissipation regime of warm inflation with nonminimal derivative coupling (70) is comparable to the high-dissipation regime of pure warm inflation (71) and the weak-dissipation regime of pure warm inflation which is given by

$$
A_{\mathrm{inf}}=-\frac{10}{3}\left(\frac{\dot{\varphi}}{H}\right)\left[\frac{V^{\prime \prime \prime}\left(\varphi_{0}\left(t_{F}\right)\right)}{3 H}\right] \text {. }
$$

\section{Conclusion}

In this paper we have studied the cosmological inflation on the nonminimal coupling where the scalar field is nonminimally coupled to gravity via its kinetic term. We have studied the warm inflationary dynamics and dissipative effects of the model. We have calculated the inflation parameters and perturbations. Next we have surveyed observational constraints on two inflationary models with a field derivative coupling to the Einstein tensor. We also have explored the evolution of the inflationary parameters in the background of the Planck + WMAP9 $+\mathrm{BAO}+\mathrm{H}_{0}$ data. For chaotic potential, as we see in Figure 1 , the values of $n_{s}, r$, and 
$\alpha_{s}$ for all given values of the number of e-folds and with $Q=1000$ lie inside the 95\% CL of the Planck + WMAP9 $+\mathrm{BAO}+\mathrm{H}_{0}$ data. Figure 2 shows that chaotic potential with $\alpha=1000$ can be compatible with the current observational data. In natural inflation with the potential $V(\varphi)=\Lambda^{4}[1+$ $\cos (\varphi / f)]$, observational constraints can be parameterized by the parameter $\gamma=f^{2} \Lambda^{4} / M^{2} M_{p}^{4}$. In Figure 3 with $\gamma=100$, the values of $n_{s}, r$, and $\alpha_{s}$ for all given values of the number of e-folds lie inside the $95 \%$ CL of the Planck + WMAP9 + $\mathrm{BAO}+\mathrm{H}_{0}$ data. Figure 4 shows that natural potential with $Q=100$ can be compatible with the current observational data. Finally, we found the non-Gaussianity in warm inflation with nonminimal derivative coupling is comparable to the high-dissipation regime of inflation and the weak-dissipation regime of inflation.

\section{Conflict of Interests}

The authors declare that there is no conflict of interests regarding the publication of this paper.

\section{References}

[1] A. R. Liddle and D. H. Lyth, Cosmological Inflation and LargeScale Structure, Cambridge University Press, 2000.

[2] J. E. Lidsey, A. R. Liddle, E. W. Kolb, E. J. Copeland, T. Barreiro, and M. Abney, "Reconstructing the inflaton potentialan overview," Reviews of Modern Physics, vol. 69, p. 373, 1997.

[3] A. Berera, "Warm inflation," Physical Review Letters, vol. 75, no. 18, pp. 3218-3221, 1995.

[4] A. Berera, M. Gleiser, and R. O. Ramos, "A first principles warm inflation model that solves the cosmological horizon and flatness problems," Physical Review Letters, vol. 83, no. 2, pp. 264-267, 1999.

[5] A. Berera, "The warm inflationary universe," Contemporary Physics, vol. 47, no. 1, pp. 33-49, 2006.

[6] I. G. Dymnikova and M. Y. Khlopov, "Self-consistent initial conditions in inflationary cosmology," Gravitation and Cosmology. Supplement, vol. 4, pp. 50-55, 1998.

[7] A. Berera and R. O. Ramos, "Absence of isentropic expansion in various inflation models," Physics Letters B: Nuclear, Elementary Particle and High-Energy Physics, vol. 607, no. 1-2, pp. 1-7, 2005.

[8] M. Bellini, "Fresh inflation: a warm inflationary model from a zero temperature initial state," Physical Review D: Particles and Fields, vol. 63, Article ID 123510, 2001.

[9] A. D. Linde, "Chaotic inflation," Physics Letters B: Nuclear, Elementary Particle and High-Energy Physics, vol. 129, no. 3-4, pp. 177-181, 1983.

[10] F. Bezrukov and M. Shaposhnikov, "The Standard Model Higgs boson as the inflaton," Physics Letters B: Nuclear, Elementary Particle and High-Energy Physics, vol. 659, no. 3, pp. 703-706, 2008.

[11] M. Atkins and X. Calmet, "On the unitarity of linearized General Relativity coupled to matter," Physics Letters B: Nuclear, Elementary Particle and High-Energy Physics, vol. 695, pp. 298302, 2011.

[12] C. P. Burgess, H. M. Lee, and M. Trott, "On Higgs inflation and naturalness," Journal of High Energy Physics, vol. 2010, no. 7, article 7, 2010.
[13] F. Bezrukov, A. Magnin, M. Shaposhnikov, and S. Sibiryakov, "Higgs inflation: consistency and generalisations," Journal of High Energy Physics, vol. 2011, no. 1, article 16, 2011.

[14] C. Germani and A. Kehagias, "New model of inflation with nonminimal derivative coupling of standard model higgs boson to gravity," Physical Review Letters, vol. 105, Article ID 011302, 2010.

[15] C. Germani and A. Kehagias, "Cosmological perturbations in the new Higgs inflation," Journal of Cosmology and Astroparticle Physics, vol. 2010, no. 5, article 19, 2010.

[16] S. Tsujikawa, "Observational tests of inflation with a field derivative coupling to gravity," Physical Review D: Particles and Fields, vol. 85, Article ID 083518, 2012.

[17] J. B. Dent, S. Dutta, E. N. Saridakis, and J.-Q. Xia, "Cosmology with non-minimal derivative couplings: perturbation analysis and observational constraints," Journal of Cosmology and Astroparticle Physics, vol. 2013, no. 11, article 58, 2013.

[18] H. M. Sadjadi and P. Goodarzi, "Reheating in non-minimal derivative coupling model," Journal of Cosmology and Astroparticle Physics, vol. 2013, no. 2, article 38, 2013.

[19] E. N. Saridakis and S. V. Sushkov, "Quintessence and phantom cosmology with nonminimal derivative coupling," Physical Review D: Particles and Fields, vol. 81, Article ID 083510, 2010.

[20] G. Gubitosi and E. V. Linder, "Purely kinetic coupled gravity," Physics Letters B: Nuclear, Elementary Particle and High-Energy Physics, vol. 703, no. 2, pp. 113-118, 2011.

[21] K. Maeda, S. Mizuno, and T. Torii, "Effective gravitational equations on a brane world with induced gravity," Physical Review D: Particles and Fields, vol. 68, Article ID 024033, 2003.

[22] M. A. Cid, S. del Campo, and R. Herrera, "Warm inflation on the brane," Journal of Cosmology and Astroparticle Physics, vol. 2007, no. 10, article 5, 2007.

[23] G. Hinshaw, D. Larson, E. Komatsu et al., "Nine-year Wilkinson Microwave Anisotropy Probe (WMAP) observations: cosmological parameter results," The Astrophysical Journal Supplement Series, vol. 208, no. 2, article 19, 2013.

[24] C. L. Bennett, D. Larson, J. L. Weiland et al., "Nine-year Wilkinson Microwave Anisotropy Probe (WMAP) observations: final maps and results," The Astrophysical Journal Supplement Series, vol. 208, no. 2, article 20, 2013.

[25] P. A. R. Ade, N. Aghanim, C. Armitage-Caplan et al., "Planck 2013 results. XXII. Constraints oninflation,” http://arxiv.org/ abs/1303.5082.

[26] J. M. Bardeen, "Gauge-invariant cosmological perturbations," Physical Review D: Particles and Fields: Particles and Fields, vol. 22, no. 8, pp. 1882-1905, 1980.

[27] E. Bertschinger, “CosmologicalDynamics," http://arxiv.org/abs/ astro-ph/9503125.

[28] K. Nozari and N. Rashidi, "Braneworld nonminimal inflation with induced gravity," Physical Review D: Particles and Fields, vol. 86, Article ID 043505, 2012.

[29] K. Nozari and N. Rashidi, "Some aspects of tachyon field cosmology," Physical Review D: Particles and Fields, vol. 88, Article ID 023519, 2013.

[30] K. Bhattacharya, S. Mohanty, and A. Nautiyal, "Enhanced polarization of the cosmic microwave background radiation from thermal gravitational waves," Physical Review Letters, vol. 97, Article ID 251301, 2006.

[31] S. Gupta, A. Berera, A. F. Heavens, and S. Matarrese, "NonGaussian signatures in the cosmic background radiation from warm inflation," Physical Review D: Particles and Fields, vol. 66, no. 4, Article ID 043510, 2002. 
[32] S. Gupta, "Dynamics and non-Gaussianity in the weakdissipative warm inflation scenario," Physical Review D: Particles and Fields, vol. 73, Article ID 083514, 2006.

[33] A. Berera, "Warm inflation in the adiabatic regime-a model, an existence proof for inflationary dynamics in quantum field theory," Nuclear Physics B, vol. 585, no. 3, pp. 666-714, 2000. 

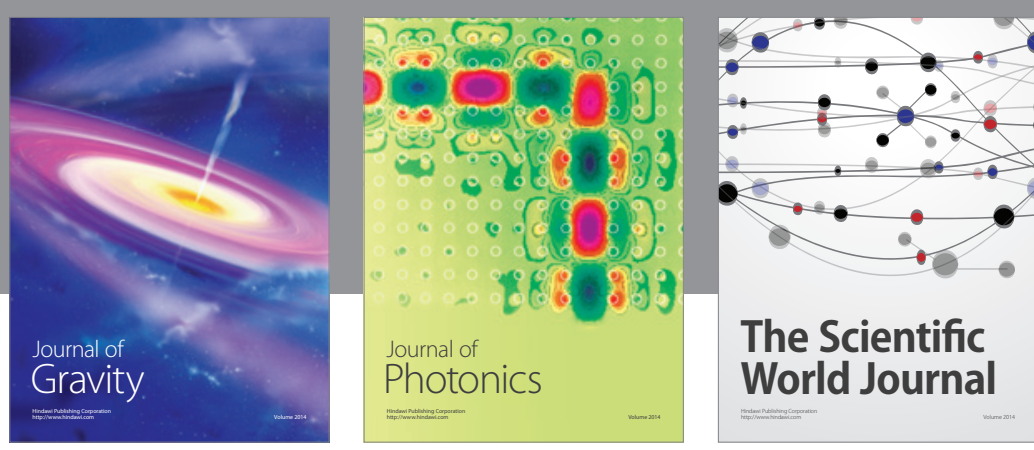

The Scientific World Journal
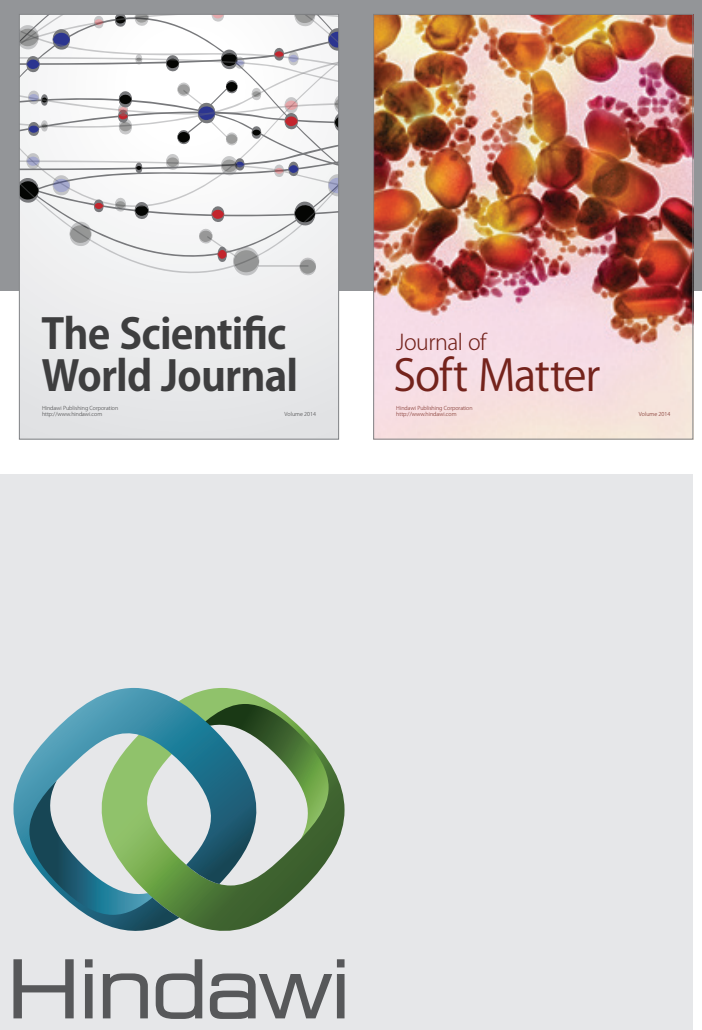

Submit your manuscripts at

http://www.hindawi.com

nternational Journal of

Statistical Mechanics
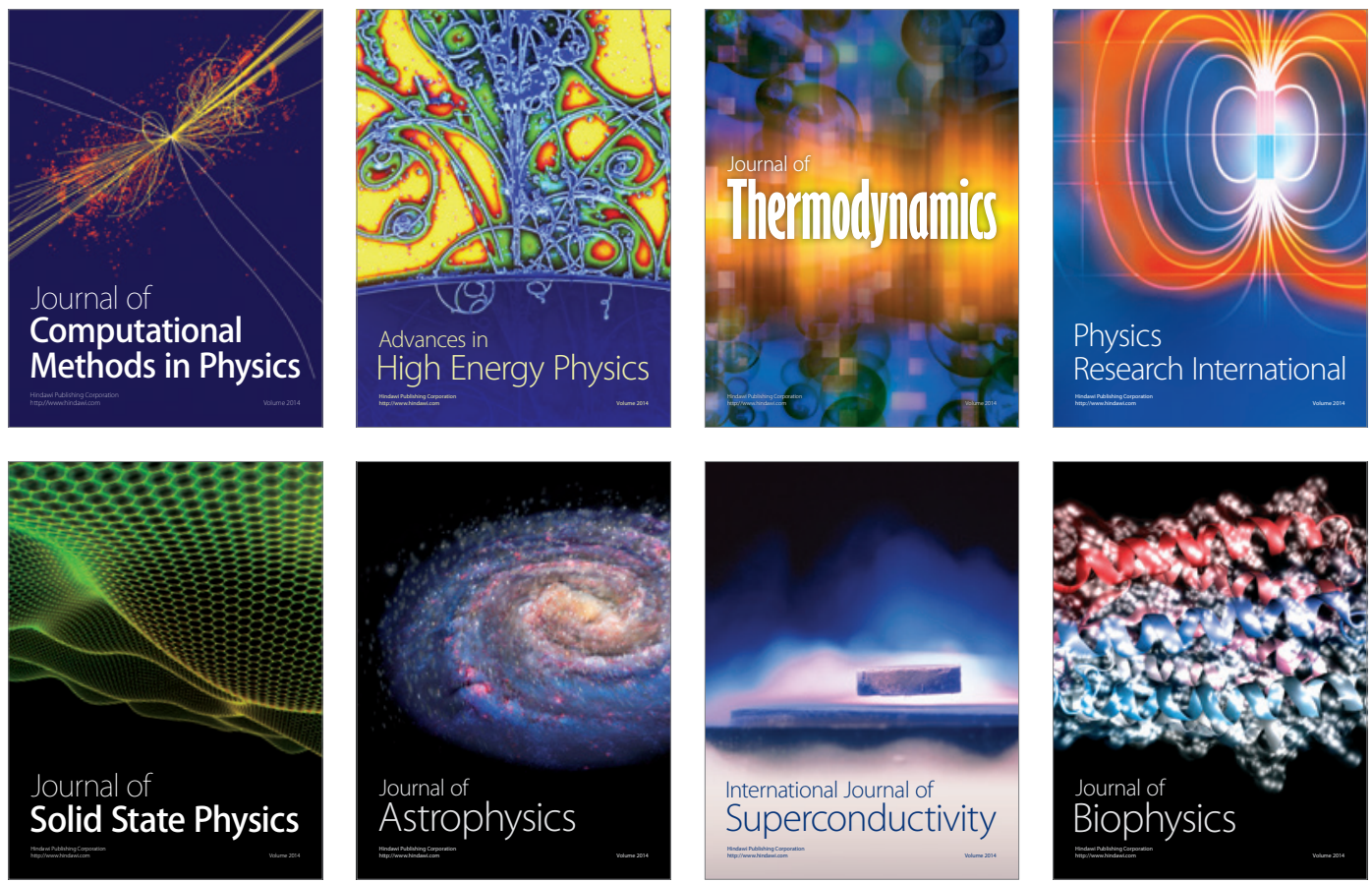
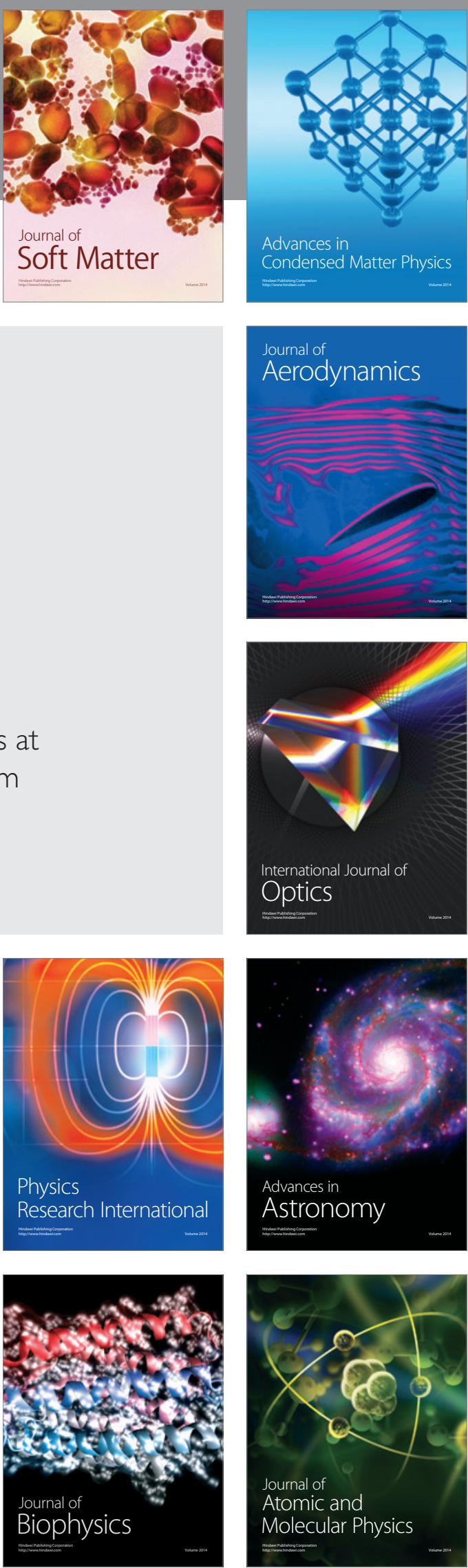